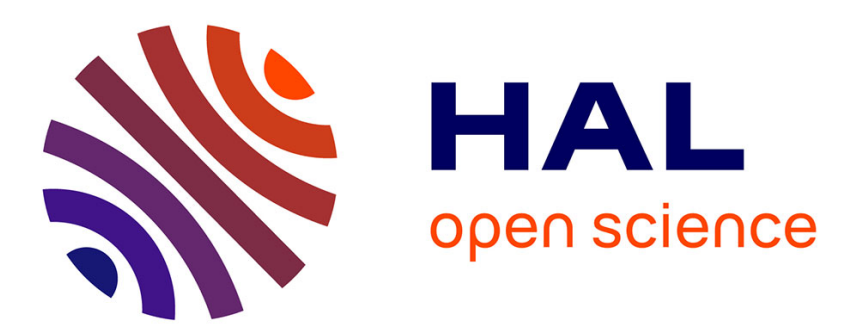

\title{
Le Logos et l'empereur, nouveaux Orphée. Postérité d'une image entrée dans la littérature avec Clément d'Alexandrie
}

Fabienne Jourdan

\section{- To cite this version:}

Fabienne Jourdan. Le Logos et l'empereur, nouveaux Orphée. Postérité d'une image entrée dans la littérature avec Clément d'Alexandrie. Vigiliae Christianae, 2008, 62 (62, 4), pp.319-333. 10.1163/157007208X247656 . hal-00392855

\section{HAL Id: hal-00392855 \\ https://hal.science/hal-00392855}

Submitted on 9 Jan 2020

HAL is a multi-disciplinary open access archive for the deposit and dissemination of scientific research documents, whether they are published or not. The documents may come from teaching and research institutions in France or abroad, or from public or private research centers.
L'archive ouverte pluridisciplinaire HAL, est destinée au dépôt et à la diffusion de documents scientifiques de niveau recherche, publiés ou non, émanant des établissements d'enseignement et de recherche français ou étrangers, des laboratoires publics ou privés. 


\title{
Le Logos et l'empereur, nouveaux Orphée «Postérité d'une image entrée dans la littérature avec Clément d'Alexandrie»
}

\author{
Fabienne Jourdan \\ CNRS_Centre Lenain de Tillemont, Paris—UMR 8167, 28 rue Serpente, 75006 Paris, France \\ jourdan.fabienne@wanadoo.fr
}

\begin{abstract}
In the Protrepticus, Clement of Alexandria suggests Orpheus' song is a prefiguration of the power of the Word. In the fourth century A. D., Eusebius and Themistius will apply this interpretation of Orpheus' song respectively to the Logos and to the Emperor. In the image proposed by Eusebius the Alexandrian's influence is obvious, whereas its transformation in Themistius illustrates its political evolution. An examination of these two different applications shows not only Clement's role in the transfer of a symbolic figure, but also the originality of its first Christian transposition in the Protrepticus.
\end{abstract}

\section{Keywords}

Orpheus, song, Christus, Clement of Alexandria, Eusebius, Themistius

Dans le Protreptique, Clément d'Alexandrie crée l'image du Christ en nouvel Orphée. Il fait d'abord passer le citharède thrace au crible de sa critique. Il prête ensuite ses traits purifiés au Seigneur qui les sublime - deux étapes qui correspondent aux deux temps caractéristiques du genre protreptique. Le catalyseur de cette opération est le Logos présenté comme «chant nouveau». Pour introduire son lecteur païen à la notion de "Parole efficace», Clément choisit en effet un mythe grec qui exalte celle-ci sous la forme du chant : les airs d'Orphée mettent en mouvement l'immobile, apaisent le sauvage ; ses vers disent et, en vertu de la métaphore grecque du "poètedémiurge», participent à l'organisation créatrice de l'univers ; ${ }^{1}$ ses incantations

1) Brisson, 2000, pp. 211-212. Voir aussi l'exégèse des vers attribués à Orphée dans le Papyrus de Derveni où les verbes évoquant l'organisation créatrice du monde ont à la fois pour sujet le poète et le Nov̂s, col. IX, 1 et col. IX, 7. Sur ce sujet, Jourdan, 2003, p. 9. 
seraient à l'origine des mystères, voire plus généralement de toutes les $\tau \varepsilon \lambda \varepsilon \tau \alpha i_{1}{ }^{2} \mathrm{Or}$, le chant n'est pas seulement musique. Il est avant tout $\lambda{ }^{\prime} \gamma o{ }^{3}{ }^{3}$ Pour confirmer le rapprochement de cette parole "orphique $»^{4}$ et du Christ,

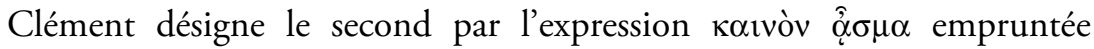
essentiellement aux Psaumes. ${ }^{5}$ Le lien entre les deux figures ainsi tissé, l'hellénisation peut avoir lieu. Elle se réalise à partir des deux facettes essentielles du citharède thrace, celle de chantre et celle de mystagogue. La transformation christique de la première donne pleinement à saisir l'action de la Parole décrite comme chant. Sous la plume protreptique, le Christ renouvelle les trois vertus du chant d'Orphée. Loin de séduire seulement animaux et pierres, il transforme par la conversion les individus plus sauvages ou pétrifiés qu'eux en hommes véritables. ${ }^{6}$ Au lieu de se contenter de chanter la naissance du monde, il la réalise et assure son maintien. ${ }^{7}$ Plutôt que d'introduire à un polythéisme idolâtre, il ouvre la porte qui mène au Dieu unique. ${ }^{8}$ Chant de métamorphose, chant démiurgique et chant théologique, la Parole assimile et dépasse ainsi les pouvoirs que la tradition

2) Voir par exemple Aristophane, Grenouilles, v. 1028 ; Horace, Art poétique, v. 396. Sur les rapports entre Orphée et les cultes païens, la bibliographie est immense. De manière générale, voir par exemple Linforth, 1941, pp. 175-261 et surtout p. 241.

3) Cette idée remonte à Platon (République, $398 \mathrm{~d}$ 3-9) selon lequel, dans le chant, l'élément essentiel est le texte ou la parole ( $\lambda$ ó $\gamma \circ \varsigma)$ que rythme et harmonie ne font qu'accompagner. Clément ne cite pas ce passage célèbre de la République, mais le connaît assurément. La suprématie de la parole dans le chant n'est d'ailleurs pas une idée qu'il emprunte uniquement à la tradition païenne. Elle correspond également à la liturgie chrétienne des premiers siècles d'où étaient bannis les instruments de musique (voir Gérold [1931] 1973 ; McKinnon, 1965 et 1994). Cette pratique se reflète dans les exégèses de Clément où tous les instruments évoqués dans la Bible sont interprétés de manière allégorique afin de ne laisser la première place qu’à la voix humaine et au Christ (cf. Péd., II, IV, 41, 4-43, 3 ; Strom. VI,

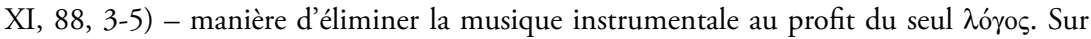
ce sujet, voir par exemple Quasten, 1930, p. 87 ; Schneider, 1999, pp. 251-252.

4) Dans cet article, nous utilisons l'adjectif "orphique» uniquement pour renvoyer au personnage d'Orphée et jamais pour évoquer la religion ou les doctrines dites "orphiques" au sens où elles remonteraient à Orphée.

5) Ps 32,$3 ; 39,4 ; 95,1 ; 97,1 ; 149,1$. Cf. Is 42,10 et Ap 14, 3. L'image renvoie au chant entonné après l'exode par Israël pour remercier Dieu de l'avoir délivré. Dans le contexte chrétien, elle fait allusion au Christ qui ouvre une ère nouvelle. Sur ce sujet, voir par exemple Brigham, 1962 ; Irwin, 1983, p. 58.

G) Protr. I, 4, 1-4.

7) Protr. I, 5, 1-4.

8) Protr. I, 10, 2-3 ; Cf. Protr. XI, 113, 4. 
grecque accorde aux airs du citharède. La méthode utilisée pour cette transformation caractérise toute la réécriture des traditions païennes entreprise dans le Protreptique. Elle comporte trois temps. Une opposition systématique à la légende hellénique évoquée ; une transposition de ses éléments qui servent alors d'expression à ceux du christianisme tout en conservant leur acception originelle ; ${ }^{9}$ leur appropriation totale enfin par laquelle ces notions passent insensiblement dans la sphère chrétienne, chargées de résonances entièrement nouvelles. ${ }^{10}$ Dans ce processus, l'allégorie n'est pas l'instrument privilégié. Clément n’invite pas à lire la vérité «logique» dans le mythe païen d'Orphée, mais emprunte à celui-ci ses traits pour évoquer la puissance du Logos. Il transpose et s'approprie la légende. L'allégorie n'intervient que dans un second temps. ${ }^{11}$

9) Le rapprochement s'opère dans cette phase par le biais de la comparaison, de la négation et de la légère transformation d'expressions qui laissent toujours transparaître la distance à franchir entre les deux sphères de croyances (un exemple de transposition peut être lu en Protr. I, 4, 1).

10) Un bon exemple d' "appropriation" chrétienne de la légende d'Orphée apparaît en Protr. XI, 114, 4. Là, les termes qui servaient à décrire le mythe grec (à savoir le thème de la métamorphose, exprimé ici par un triple emploi du préverbe $\mu \varepsilon \tau \alpha ́$ et surtout par le verbe

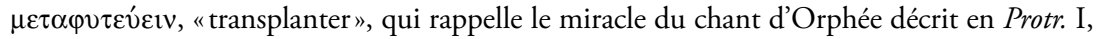
$1,1)$ permettent d'évoquer la puissance christique sans qu'il soit fait la moindre allusion directe à la source païenne. - Il faut ajouter que dans ces trois moments (opposition/transposition/appropriation), Clément recourt toujours au même outil : la polysémie, qui lui permet de faire résonner un mot dans chacun des deux idiolectes, païen et chrétien. Il use alors essentiellement de deux figures polysémiques : la syllepse sémantique (qui crée l'ambiguïté au sein d'un même mot exprimé une seule fois) et l'antanaclase (qui produit une alternance en répétant plusieurs fois le mot lequel, à chaque occurrence, reçoit un sens distinct).

11) Nous souhaitons ici simplement mettre en garde contre une évocation peut-être trop rapide de la méthode allégorique que Clément emploierait à propos du mythe d'Orphée dans le Protreptique (voir Tabaglio, 1999, p. 80 et Rœssli, 2002, p. 507). Mis à part l'interprétation de l'écriture égyptienne dans le cinquième Stromate, l'Alexandrin n'applique l'allégorie qu'à l'Écriture et jamais aux mythes païens. Ici, il commence par transposer la légende en disant que, supérieur à Orphée, le Christ apaise les animaux les plus sauvages que sont les hommes. L'allégorie apparaît donc après la transposition, à un deuxième niveau qui correspond à l'assimilation chrétienne de la légende. Elle consiste seulement dans le fait de voir des pécheurs dans les animaux séduits par le chant nouveau. Or, il faut bien reconnaître que, plus que d'une allégorie, il s'agit avant tout d'une métaphore lexicalisée ou du moins héritée directement des traditions philosophiques et religieuses, l'association des hommes en proie aux vices aux diverses bêtes sauvages étant fréquente d'une part chez Platon ou les Stoïciens et d'autre part dans l'Ancien Testament ou dans les bestiaires de 
L'image du Christ, nouvel Orphée, ainsi créée a-t-elle eu une postérité ? Le cas échéant, la méthode mise en œuvre par Clément a-t-elle été reprise ? Ses successeurs ont-il fait preuve de semblable génie d'invention ?

Le chant de métamorphose entonné dans le Protreptique n'a éveillé que deux échos. Au IV siècle après J.-C., Eusèbe et Themistios appliquent communément au Logos et à l'empereur l'interprétation clémentine du chant d'Orphée comme préfiguration des pouvoirs de la Parole. La reprise directe de l'image par le premier permet de saisir directement l'influence de l'Alexandrin dans la création de celle-ci. Sa transformation chez le second (bien qu'il soit impossible de prouver qu'il a lu le Protreptique) fournit un exemple de son devenir politique. L'étude de l'une et de l'autre fera apparaître non seulement le rôle de Clément dans le transfert d'une figure symbolique, mais aussi l'originalité de la première transposition chrétienne de cette figure dans le Protreptique.

\section{Le Logos, nouvel Orphée chez Eusèbe}

Eusèbe imprime une simplification à la comparaison entre le Logos et Orphée créée dans le Protreptique. Par là, il confirme la pertinence de son choix pour dispenser un enseignement chrétien à des païens. ${ }^{12} \mathrm{Il}$ sanctionne l'intuition de Clément et assure son succès.

Dans la seconde partie du discours d'éloge de Constantin rédigé à l'occasion du trentième anniversaire de son règne (en 336), ${ }^{13}$ l'évêque de Césarée propose une initiation à la doctrine chrétienne. Son exposé est en lien direct au panégyrique. L'exercice de la royauté terrestre étant conçu par lui à l'image de celle exercée dans l'univers par le $\operatorname{Logos}^{14}$ (elle-même à

l'époque comme le Philologos. (La seule originalité de Clément consiste peut-être à christianiser cette image en la reliant au mythe d'Orphée et en voyant dans les animaux des pécheurs.) Même si l'Alexandrin emploie le verbe caractéristique de l'allégorie ( $\alpha i v i ́ \sigma \sigma o \mu \alpha \imath$ ) dans la suite de son rapprochement (Protr. I, 4, 3), il l'utilise pour commenter un texte biblique $(\mathrm{Mt}, 7,15)$ et non plus directement le mythe. Il ne lit donc pas allégoriquement dans la légende d'Orphée une représentation "voilée» des miracles christiques.

12) Pour d'autres comparaisons entre les textes de Clément et d'Eusèbe, voir Eisler, [1925] 1966, pp. 61-71 ; Friedman, [1970] 1999, pp. 58-63 ; Naldini, 1993, p. 335 ; et surtout Roessli, 2002.

13) Voir la préface à la traduction du texte par P. Maraval, 2001, p. 24.

14) Sur cette théorie telle qu'elle est développée chez Eusèbe, Hadot, 1972, col. 614-615. Sur ses origines, Ricken, 1967. Fr. Ricken met en évidence que, chez Eusèbe, la doctrine du Logos, intermédiaire cosmique entre Dieu qui est transcendant et le monde en devenir, est 
l'image de celle du Père), ${ }^{15}$ le roi humain doit diriger son empire à l'imitation du Logos (I, 6). L’enseignement théologique est donc considéré comme un préalable indispensable à la définition des vertus de l'homme politique. S’adressant à un public composé à la fois de païens et de chrétiens, Eusèbe choisit comme Clément de parler le langage familier aux premiers. Il emploie ainsi la notion philosophique de Logos plutôt que celle de Christ et, pour justifier l'Incarnation, renvoie à l'image d'Orphée :

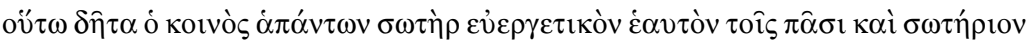

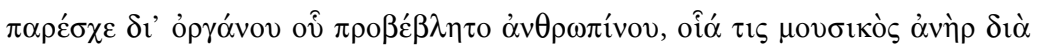

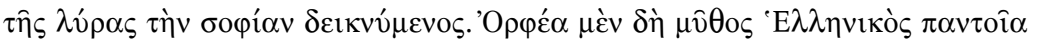

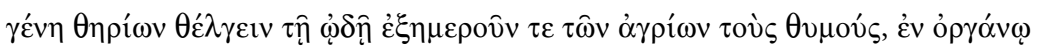

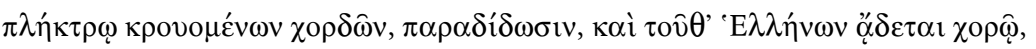

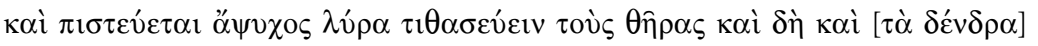

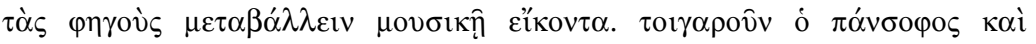

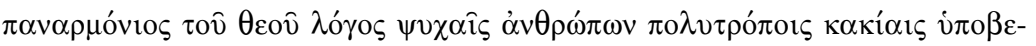

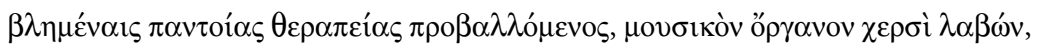

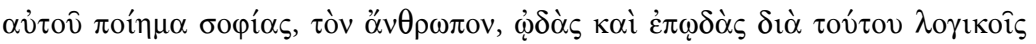

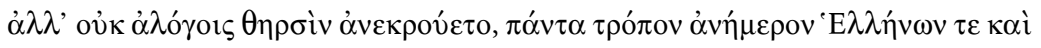

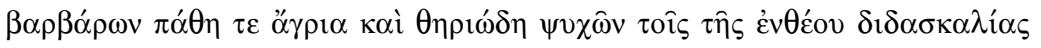

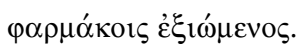

Ainsi donc, le sauveur commun d'absolument tous les hommes s'est donné luimême comme bienfaiteur et sauveur pour tous par l'intermédiaire de l'instrument humain qu'il a proposé à la vue, comme un musicien qui montre sa science grâce à sa lyre. (5.) Le mythe grec transmet ainsi qu'Orphée charmait toutes sortes de bêtes sauvages par son chant et domptait les fureurs des animaux féroces en frappant du plectre les cordes de son instrument. Cela est chanté en chœur par les Grecs et l'on croit qu'une lyre sans âme apprivoisait les bêtes sauvages et faisait même se déplacer les arbres, les chênes, qui cédaient à la musique. C'est pourquoi le Logos de Dieu, sage en toutes choses et en tout point harmonieux, proposant toutes sortes de soins aux âmes des hommes soumises à des maux en tout genre, a pris dans ses mains l'instrument de musique, la création de sa sagesse, l'homme. Il a commencé à jouer grâce à lui, des odes et épodes pour les êtres raisonnables et non pour les bêtes dépourvues de raison, chassant, par les remèdes de l'enseignement

empruntée non au stoïcisme mais (directement ou, si indirectement, par le biais de Philon, d'Origène ou de Numénios) au (médio-)platonisme. Sa conception est en effet à rapprocher de celle de l'âme du monde propre à cette école (Ricken, 1967, pp. 348-354 et p. 357).

15) Le Père règne, mais ne gouverne pas. Sa royauté est exercée par le Fils, le Logos. Sur ce sujet, voir la préface de P. Maraval, 2001, pp. 52-54. 
divin, toute manière grossière des Grecs et Barbares ainsi que les passions féroces et sauvages de leurs âmes (II, 14, 4-5). ${ }^{16}$

Il est possible de lire ici comment le déplacement thématique de l'image créée par Clément, associé à une reprise lexicale de son expression dans le Protreptique et à une simplification, confirme l'entrée de celle-ci dans la tradition chrétienne. ${ }^{17}$

Eusèbe n'utilise certes pas la figure d'Orphée pour transmettre le même message que son prédécesseur. Dans ce passage, il explique que le Logos s'est incarné dans le corps, considéré comme un instrument, pour se révéler aux hommes. ${ }^{18}$ Le point de comparaison avec le citharède thrace réside alors dans l'emploi de la lyre (ce qui confirme l'association privilégiée de cet instrument à Orphée dans l'imaginaire chrétien également). L'évêque

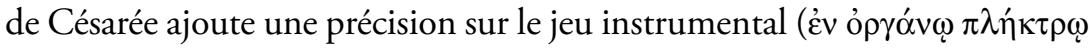

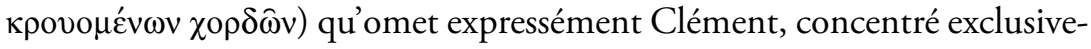
ment sur le chant, conçu par lui comme parole et donc élément fondamental de la comparaison entre Orphée et le Logos. C'est précisément parce qu'il n'a plus besoin de justifier l'association de ces deux figures qu'Eusèbe peut déplacer le motif du rapprochement - ce qui prouve rétrospectivement que la proposition de Clément a été reçue et est ancrée dans l'esprit du chrétien. ${ }^{19}$

Malgré cet écart thématique, l'emprunt au Protreptique apparait ici avec évidence dans l'identité du vocabulaire. Le cour de la légende, l'instrument et les chants sont évoqués dans des termes proches ou identiques. ${ }^{20}$ Le Logos est qualifié de manière semblable à quelques décalages près. ${ }^{21}$ Mais

16) Nous traduisons.

17) Une influence de l'iconographie du Christ sous les traits d'Orphée qui se développe aux $\mathrm{III}^{\mathrm{e}}$ et IV $\mathrm{V}^{\mathrm{e}}$ siècles ne serait certes pas à exclure. Mais le texte d'Eusèbe est quoi qu'il en soit directement inspiré du Protreptique.

18) L'image de l'âme considérée comme une lyre dont les cordes représenteraient le corps est proposée dans le Phédon (85 e) par Simmias, élève du pythagoricien Philolaos. Elle est ici détournée en ce qu'Eusèbe ne s'intéresse qu'au corps dans lequel s'est incarné le Christ.

19) On peut aussi remarquer avec R. Skeris que Clément reste fidèle à la pensée sémitique en traitant toujours la notion de parole en lien avec celle de dabar, de parole efficace. Insistant moins sur cet aspect, Eusèbe s'éloigne de la notion juive pour s'approcher davantage du concept grec de $\lambda$ ó ${ }^{\circ}$ (Skeris, 1976, p. 159).

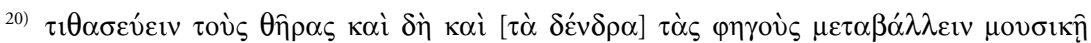

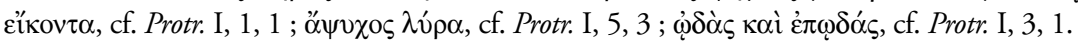

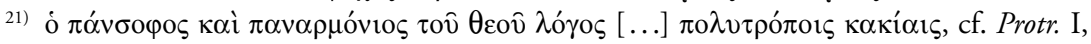

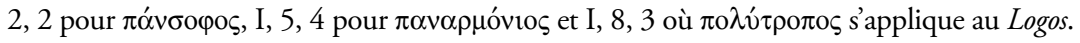


Eusèbe condense son discours et par là, assume pleinement la comparaison instaurée par l'Alexandrin. Il unit d'abord, en traitant la question du corps comme instrument de la révélation, deux thèmes distincts chez Clément : celui du charme exercé sur les animaux et donc sur les passions, et celui de la création harmonieuse de l'univers où intervient la notion d'homme,

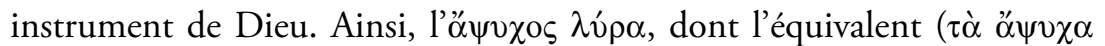
óp $\alpha v \alpha$ ), dans le Protreptique, ${ }^{22}$ apparaît au moment de l'exposé démiurgique, est ici rattaché au mythe de la séduction des bêtes. Eusèbe simplifie ensuite l'image païenne donnée dans l'incipit du traité de Clément en en détournant le propos. La chanson populaire grecque relative à Amphion et Arion est ici évoquée à propos d'Orphée sur lequel seul se concentre la comparaison. Eusèbe supprime même la référence au "chant nouveau » et

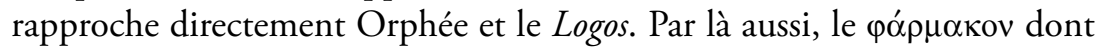
parle Clément ${ }^{23}$ n'est plus le chant, mais, directement, l'enseignement chrétien. L'évêque adopte alors plus clairement la structure allégorique, selon une construction qui rappelle l'interprétation morale de la légende par Macrobe. ${ }^{24}$ Il commence par évoquer le mythe avant de donner son

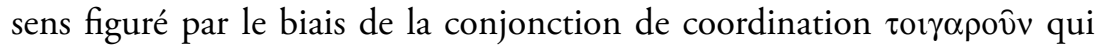
introduit l'explication. Dans le Protreptique, Clément ne propose pas un passage aussi franc de la tradition païenne à l'enseignement chrétien. Eusèbe ne joue plus sur les sous-entendus. Les termes employés à double entente par Clément sont devenus propriété de l'idiolecte chrétien :

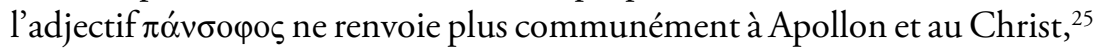
comme dans le Protreptique, mais seulement au second ; le passage des termes de la légende dans la sphère chrétienne est à ce point accepté

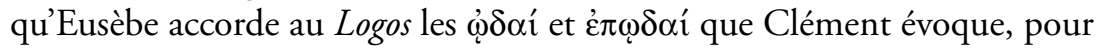

\footnotetext{
22) I, 5, 3.

23) Protr. I, 2, 4.

24) Macrobe, Commentaire au Songe de Scipion, II, 3, 8 : Hinc aestimo et Orphei vel Amphionis fabulam, quorum alter animalia ratione carentia, alter saxa quoque trahere cantibus ferebantur, sumpsisse principium, quia primi forte gentes vel sine rationis cultu barbaras, vel saxi instar nullo affectu molles, ad sensum voluptatis canendo traxerunt, "Telle est à mon sens l'origine des mythes d'Orphée ou d'Amphion, qui, par leurs chants, amenaient à eux, diton, l'un, les animaux privés de raison, l'autre, les rochers aussi : il se peut qu'ils aient été les premiers à avoir par leur chant amené à un sentiment de plaisir des peuples barbares étrangers à l'usage de la raison, ou bien, à l'instar du roc, rebelles à toute émotion (trad. de M. Armisen-Marchetti).» Chez l'exégète latin, le quia permet de passer du sens littéral au sens allégorique.
}

25) Protr. I, I, 2. 
les critiquer, à propos des citharèdes païens. ${ }^{26}$ L'opposition entre les auditeurs d'Orphée et du Logos, martelée par Clément, est ici parfaite par

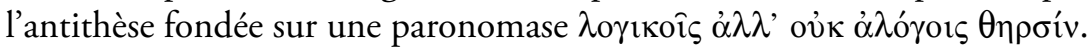
Par cette figure qui relève d'une assimilation du style même de l'Alexandrin, ${ }^{27}$ Eusèbe révèle de manière synthétique le fond de l'antagonisme que voulait faire apparaitre son modèle. C'est parce qu'il réécrit, là où Clément est pris dans le bouillonnement enthousiaste de la création, qu'il peut rendre l'image dont il hérite avec une telle clarté. L'opération s'achève dans la transformation, sous sa plume, de l'allégorie utilisée dans un second temps par l'Alexandrin en simple métaphore. Là où, après avoir approprié la légende orphique à sa peinture du Logos, Clément associe chaque type de pécheur à un animal incarnant la passion de celui-ci, Eusèbe applique sim-

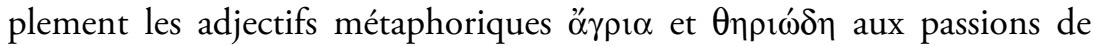
l'âme qu'il nomme explicitement $(\pi \dot{\alpha} \theta \eta \psi v v \chi \omega \hat{\omega} v)$. Cette atténuation de l'image assure son devenir topique.

En détournant légèrement de son propos la comparaison entre Orphée et le Logos inaugurée par Clément, en la condensant et la simplifiant, Eusèbe l'assume pleinement. Contrairement à son prédécesseur, il réalise complètement l'interprétation allégorique du mythe avant de la transformer en métaphore sur le point de se figer ; plus clairement que lui, il exprime le fond de l'opposition entre l'œuvre d'Orphée et celle du Logos. Son texte entérine l'image proposée par Clément. Mais, par ce qu'il supprime, il nous fait rétrospectivement découvrir ce qui nourrit l'originalité de la représentation du Christ en nouvel Orphée dans le Protreptique : un dialogue constant et ambigu avec les païens où se mêlent opposition et souci d'exhortation ; le désir de montrer la supériorité du Christ qui conduit à exalter les pouvoirs orphiques du Logos et permet de détacher alors l'image de son modèle pour lui donner un épanouissement libre ; et surtout le motif du chant comme expression figurée la plus achevée de l'efficace du Logos. Chez Clément, l'Orphée païen disparaît dans la condamnation pour que puisse se produire réellement sa transfiguration. Advient alors sa nouvelle figure, le chantre à la puissance de métamorphose régénératrice synonyme de conversion. ${ }^{28}$ Chez Eusèbe, la comparaison reste limitée à ce paragraphe tandis qu'elle s'épanouit à travers tout le Protreptique. Le Logos

\footnotetext{
26) Protr. I, 3, 1.

27) Voir par exemple, Protr., I, 10, 3. Sur le jeu de Clément à partir du mot $\lambda$ ó $\gamma o \varsigma$ et de ses dérivés, voir Mondésert, 1954.

28) Cf. Protr., I, 4, 1-4.
} 
prend cette fois au poète ses chants et leurs vertus métaphoriques, mais ne se déploie pas en nouvel Orphée. L'allégorie entièrement assumée a l'effet paradoxal de permettre une transposition plus affirmée, mais d'empêcher à l'image de se perpétuer hors de son cadre, c'est-à-dire d'empêcher sa complète appropriation. Dans sa fougue créatrice étrangère à un exposé si formel, Clément seul a laissé le nouvel Orphée chanter librement un air véritablement nouveau.

\section{L'empereur, nouvel Orphée, chez Themistios}

Le païen Themistios (317-388) utilise alors la légende presque à la manière de Clément en ce qu'il fait encore surgir un nouvel Orphée, cette fois en la personne de l'empereur. Il est cependant difficile d'établir la dépendance de l'orateur au Protreptique. On peut certes aisément concevoir que, pour préparer ses discours d'éloge ou de remerciement aux empereurs et en particulier à Constantin, Themistios a lu le texte d'Eusèbe, composé à l'occasion du trentième anniversaire. Ses réflexions politiques semblent même le confirmer. ${ }^{29}$ Établir sa dépendance au Protreptique est en revanche moins évident. Trois hypothèses se présentent. Il est possible d'imaginer que ce païen qui entretient d'excellentes relations avec les chrétiens a eu directement accès à l'ouvrage. Celui-ci lui aurait servi dans son rôle d'intermédiaire conciliant entre les deux partis. Son développement sur Orphée et l'empereur, ancré dans une réflexion sur le pouvoir de la parole, peut néanmoins aussi relever de sa propre interprétation, en tant que rhéteur, du discours d'Eusèbe (lui-même dépendant du Protreptique). Il y aurait dans ce cas un rapport indirect à Clément. Dans le choix d'une comparaison flatteuse pour l'empereur, Themistios peut enfin avoir de luimême conçu ce développement, la réflexion sur le logos étant d'actualité à une époque où le débat entre païens et chrétiens n'est pas achevé, parce qu'elle permet la rencontre spirituelle entre les deux univers de pensée. ${ }^{30}$

\footnotetext{
29) Sur la théorie politique de Themistios, voir Dagron, 1968.

30) C'est précisément le thème qui anime tout le Protreptique. Il faut avoir conscience que, dans deux situations spirituelles relativement proches et à partir d'un même matériau légendaire, deux hommes peuvent développer une même idée sans nécessairement avoir communiqué entre eux. Un tel phénomène relève de la parenté des structures de l'imaginaire. Il n'en demeure pas moins que Clément a certainement rendu plus courantes les réflexions sur le pouvoir de la parole comparée au chant d'Orphée. Le développement de Themistios peut ainsi lui être, même lointainement et inconsciemment, redevable.
} 
Dans ce cas, bien que peu probable, une influence de l'iconographie n'est pas tout à fait à exclure. ${ }^{31}$

Quoi qu'il en soit, ${ }^{32}$ dans un discours d'éloge de Constantin II, Themistios adopte le même type de présentation que le Protreptique. Il passe d'une évocation admiratrice des prodiges du citharède au démenti de son œuvre civilisatrice. Là où Clément dénonce l'instaurateur de l'idolâtrie qui perd les hommes, l'orateur interprète le meurtre de celui-ci par les femmes comme l'échec de son action apaisante sur les êtres humains : le poète ne serait parvenu qu'à "ensauvager" les Thraces. Après ce tableau nuancé, Themistios exalte par contraste la puissance du roi vainqueur des Barbares en des termes empruntés à la version élogieuse de la légende, à la manière de ce que fait l'Alexandrin dans sa présentation du Logos. Or, comme dans le Protreptique, bien que dans le registre cette fois politique, le motif de la comparaison est l'efficace de la parole :

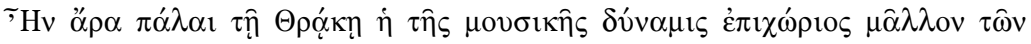

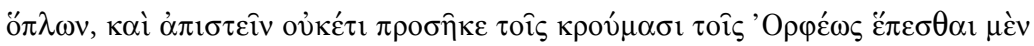

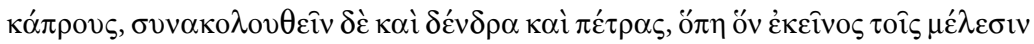

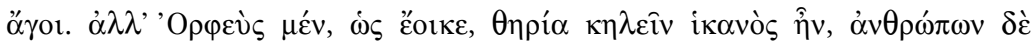

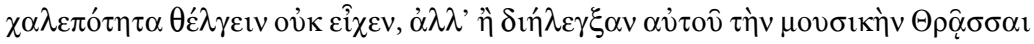

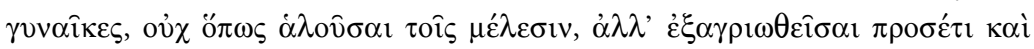

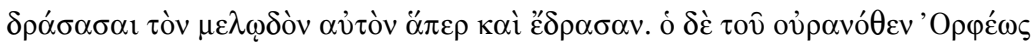

31) Aux III ${ }^{e}$ et $\mathrm{IV}^{e}$ siècles, en effet, se développe la peinture Christ sous les traits d'Orphée. Cette iconographie est cependant limitée à quelques documents de l'empire romain occidental et relève surtout du symbolisme de l'âge d'or (sur ce sujet voir par exemple Vieillefon, 2003). Or, Themistios joue quant à lui sur le thème de la domestication par le pouvoir de la parole qui est proprement littéraire : il rappelle le Protreptique, mais peut aussi être issu d'une image empruntée à des rhéteurs païens dont Themistios aurait, en bon orateur, fréquenté les ouvrages (Quintilien ou Macrobe évoquent Orphée pour exalter certes le pouvoir de la musique, voir Institution oratoire, I, X, 9 et Commentaire au Songe de Scipion, II, 3,8 , mais musique et paroles sont très proches dans l'Antiquité puisque la première comprend tous les arts des Muses. Themistios unit également les deux dans son texte, même si, chez lui le rapprochement est peut-être inspiré par l'image de Clément. L'efficacité persuasive de la parole d'Orphée est encore soulignée chez les païens par Eschyle, Agamemnon, v. 1629-1630 ; Euripide, Iphigénie à Aulis, v. 1211, Alceste, 357 et Héraclite, Histoires incroyables XXIII).

32) Il est impossible de trancher le débat sur la dépendance du discours de Themistios au Protreptique. Nous constatons simplement les affinités entre les deux textes qui semblent trahir une influence, même indirecte et lointaine, de l'image créée par Clément. 


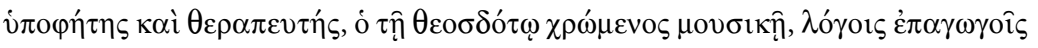

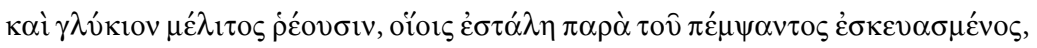

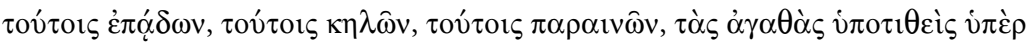

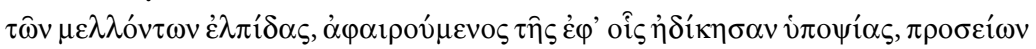

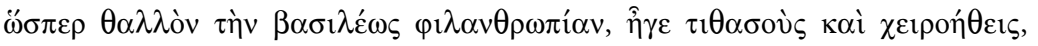

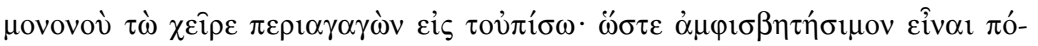

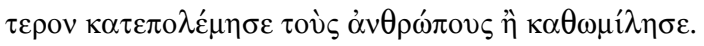

C'est qu'autrefois, en Thrace, la coutume du pays était de considérer davantage la puissance de la musique que celle des armes et il ne convenait plus de douter que les sangliers suivaient les airs produits par Orphée sur sa lyre, et que les arbres et les pierres l'accompagnaient quand il les menait par ses chants. Mais Orphée, à ce qu'il semble, bien que capable d'apaiser les bêtes sauvages, ne parvenait à charmer la méchanceté des humains. Les femmes thraces apportèrent le démenti à l'effet de sa musique parce qu'au lieu de se laisser prendre par ses chants, elles devinrent encore plus sauvages et firent au chanteur ce qu'elles lui firent. Mais lui, l'interprète et serviteur de l'Orphée céleste, usant de la musique donnée par Dieu, par ses paroles engageantes qui répandaient la douceur du miel, avec lesquelles l'avait mandé et dont l'avait pourvu celui qui l'avait envoyé, il enchantait, apaisait, encourageait. Il fit naître de bons espoirs pour l'avenir. Chassant le soupçon qui pesait sur les coupables et déployant devant eux, comme on agite une branche, ${ }^{33}$ sa philanthropie de roi, par ses paroles, il les menait apprivoisés et dociles, leur amenant presque les mains derrière le dos, si bien que l'on pouvait se demander s'il faisait la guerre aux hommes ou s'il se le conciliait (Discours XVI, 209 c-d = OT $\left.35 \operatorname{Kern}^{34}\right){ }^{35}$

Themistios semble ici unir les pensées de Clément et d'Eusèbe pour donner un portrait du roi vainqueur des Barbares. Il adopte la théologie politique du second qui fait du roi l'imitateur et le serviteur de Dieu sur terre. ${ }^{36}$ La figure d'Orphée sert ainsi doublement. Elle donne une image de Dieu (ou du Christ-Logos - Themistios, qui n'est pas chrétien, reste volontairement imprécis), puisque l'orateur parle de l' "Orphée céleste» dont l'empereur est l'interprète et le serviteur. Puis, par application de la théorie

\footnotetext{
33) Il s'agit ici de l'image de la branche que l'on agite comme un appât pour attirer un animal ou au contraire pour l'effrayer.

34) Dans le deuxième tome de l'édition des fragments orphiques de A. Bernabé qui va paraitre dans la Bibliotheca Teubneriana, ce témoignage correspondra aux textes n 921, 954 III, 1051, 1078 II. Je remercie A. Bernabé de m’avoir communiqué sa table de correspondance.

35) Nous traduisons.

36) Sur la conception de l'empereur comme imitateur sur terre de la philanthropie de Dieu telle qu'elle est développée chez Themistios, Hadot, 1972, col. 603-604. Sur la théorie politique de Themistios, Dagron, 1968.
} 
d'Eusèbe, dont l'utilisation de la figure d'Orphée est restée à ce premier stade, elle fournit un modèle pour décrire l'activité royale qui imite celle de Dieu $^{37}$ et, de manière sous-entendue, du Logos : par ses $\lambda$ ófor précisément, qui sont assimilés à la musique donnée par Dieu, l'empereur exercerait le

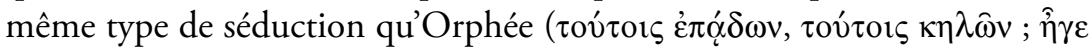
$\tau \imath \theta \alpha \sigma o v ́ \varsigma) .{ }^{38}$ C'est ici que Themistios semble rejoindre l'inspiration de Clément. Non seulement il fait de la parole le véhicule qui permet de passer du personnage légendaire à Dieu (lequel gratifie le roi de cette parole), puis à l'empereur; mais, d'une certaine façon, il prolonge l'entreprise de l'Alexandrin en donnant naissance à son tour à un nouvel Orphée, et ce par l'invitation à appliquer complètement la théorie politique suggérée par Eusèbe.

Outre le même emploi du motif de la parole, il est alors possible de rapprocher le texte de Themistios de l'œuvre créatrice de l'Alexandrin pour trois raisons.

1. Après une présentation trahissant l'admiration, l'orateur réécrit le portrait classique d'Orphée de manière critique pour le faire servir à un éloge, qui se nourrit à la fois de l'opposition et de la transposition de l'œuvre du citharède (les femmes s'ensauvagent puis les Barbares sont domptés).

2. Il ne s'adonne pas à une interprétation allégorique explicite qui cloisonnerait les domaines : cela lui permet de sanctionner l'image de Dieu ou du Christ en nouvel Orphée par la désignation directe du premier avec le nom du second.

3. Il crée enfin un successeur à cette figure, lequel semble pouvoir s'épanouir librement comme le montre le développement final de l'image où l'appropriation du mythe est totale. ${ }^{39}$

37) On peut aussi penser à une application lointaine de la théorie platonicienne de l'homme royal. Mais s'adressant à l'empereur chrétien, Themistios use sans doute de cette doctrine telle qu'elle a été revue par les coreligionnaires de celui-ci comme Eusèbe.

38) La mention de la "philanthropie royale" rappelle aussi nettement une caractéristique accordée généralement au Christ dans la littérature chrétienne.

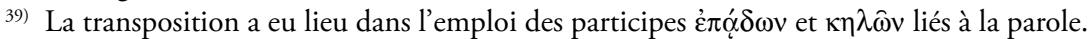

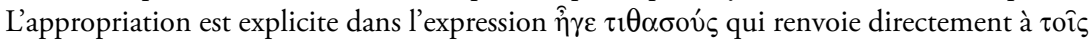
$\mu \varepsilon ́ \lambda \varepsilon \sigma ı$ ó $\gamma o t$. Il y a appropriation parce que tout en renvoyant formellement à la légende, l'expression s'en détache progressivement pour évoquer ici la conciliation de l'ennemi. La dernière proposition sanctionne ce processus : après le tableau qui a précédé, on comprend que c'est en nouvel Orphée que l'empereur a obtenu cette pacification des relations. 
L'imaginaire créateur de Themistios ne s'est peut-être pas développé directement à partir du Protreptique. Son rapprochement entre Orphée et l'empereur a pu s'élaborer par le biais d'une influence indirecte de cet ouvrage, grâce à une lecture de l'éloge d'Eusèbe à Constantin par exemple ; voire indépendamment de lui, au cours d'une réflexion sur un thème pouvant réunir les pensées païenne et chrétienne. Pourtant, le traitement de l'image chez Clément semble être, même lointainement, à l'origine de celui de l'orateur. Il a pu marquer les esprits ${ }^{40}$ et agir ainsi inconsciemment sur ce dernier. Il est alors remarquable que ce soit peut-être à partir de la version christique d'Orphée qu'un païen donne naissance à un nouvel Orphée - lequel cependant, même considéré dans son rôle politique, est encore un chrétien. Clément a engendré un modèle fécond en métamorphoses.

Dans le Protreptique, Clément d'Alexandrie s'approprie la légende d'Orphée citharède et représente le Logos-Christ en nouveau chantre thrace aux pouvoirs sublimés. Son intuition connaît deux développements littéraires. Eusèbe en hérite directement et, par sa présentation à la fois synthétique et concise, la fait entrer dans la tradition. Maintenant les deux niveaux, païen et chrétien, de l'allégorie, il propose cependant une image moins exaltée que celle de son prédécesseur. ${ }^{41}$ L’orateur Themistios prolonge ensuite, directement ou non, l'œuvre inaugurée par l'Alexandrin. Il se souvient peut-être de l'assimilation du Christ à Orphée, proposée dans le Protreptique, ou la conçoit de nouveau à partir par exemple de sa lecture d'Eusèbe (qui est influencé par Clément), voire de sa propre réflexion sur une image païenne que le christianisme est en train de s'approprier dans sa littérature comme dans son iconographie. Il l'emploie alors pour mener à terme la théologie politique de l'évêque de Césarée, qui ne s'était peut-être

40) Le Protreptique a été lu par les chrétiens, ne serait-ce que par Eusèbe que connaît sans doute Themistios. La méthode par laquelle l'Alexandrin opère l'hellénisation du Christ sous les traits d'Orphée (par le triple processus d'opposition, de transposition et d'appropriation de la légende) a aussi pu être empruntée aux écoles de rhétorique ou être réutilisée par celles-ci ensuite. Le cas échéant, même lointainement, le Protreptique peut avoir influencé l'écriture de Themistios.

41) Nous nous opposons par là aux conclusions de J. B. Friedmann ([1970] trad. fr. 1999, pp. 62-63 ; voir aussi Rœessli, 2002) proposant de lire chez Eusèbe une image plus favorable à Orphée que chez Clément. L'Alexandrin passe certes le citharède au purgatoire de sa critique, mais c'est pour pouvoir mieux appliquer les traits sublimés de celui-ci au Christ, Orphée d'un genre nouveau qui convertit même son modèle païen. 
pas exprimé si clairement, faisant par là advenir un nouvel Orphée en l'empereur. Au cours de ce processus, il recourt à la notion de parole, familière à Clément, qu'Eusèbe a abandonnée au profit de celle de l'instrument, et adopte une écriture de la transformation qui rappelle celle mise en œuvre dans le Protreptique. C'est ainsi vraisemblablement en passant par l'Orphée christique que, sous la plume d'un païen, advient un Orphée «impérial». Par ses résonances lointaines, le Protreptique se révèle être le point de départ des métamorphoses théologico-politiques du citharède thrace.

\section{Bibliographie}

Brigham, F. H., 1962, "The Concept of the New Song in Clement of Alexandria’s Exhortation to the Greeks", in $C F$ 16, pp. 9-13.

Brisson, L., 2000, «Le Discours comme univers et l'univers comme discours», in Brisson, L., Lectures de Platon, Vrin («Histoire de la philosophie»), pp. 209-218.

Dagron, G., 1968, L'Empire romain d'Orient au IV siècle et les traditions politiques de l'hellénisme, «Le témoignage de Themistios», De Boccard ("Travaux et Mémoires du centre d'histoire et de civilisation de Byzance" 3), Paris.

Eisler, R., [1925] 1966², Orphisch-dionysische Mysteriengedanken in der christlichen Antike, Georg OlmsVerlagsbuchhandlung, Hildesheim. (Reprografischer Nachdruck der Ausgabe Leipzig und Berlin 1925).

Friedman, J. Block, [1970] 1999, Orpheus in the Middle Ages, Harvard University Press, Cambridge (Mass.). Trad. fr. Orphée au Moyen Âge, par J.-M. Roessli, avec le concours de V. Cordonier et F. X. Putallaz, Cerf ( Vestigia» n 25), Paris-Fribourg, 1999, avec postface de J. M. Roessli).

Gérold, Th., [1931] 1973, Les Pères de l'Église et la musique, [Strasbourg], Minkoff reprint, Genève.

Hadot, P., 1972, article «Fürstenspiegel», in RAC 8, col. 555-632.

Irwin, E., 1982, "The Songs of Orpheus and the New Song of Christ", in J. Warden (ed.), Orpheus, "The metamorphoses of a myth", University of Toronto Press, TorontoBuffalo-London, pp. 51-62.

Jourdan, F., 2003, Le Papyrus de Derveni, Les Belles Lettres, ("Vérité des mythes»), Paris.

Maraval, P., 2001, Eusèbe de Césarée. La théologie politique de l'Empire chrétien - Louanges de Constantin, introduction, traduction et notes, Cerf ("Sagesses chrétiennes»), Paris.

McKinnon, J., -1965, "The Meaning of the Patristic Polemic against Musical Instruments», in Current Musicology 1., New York, pp. 68-82. Réimprimé dans McKinnon, J., The Temple, the Church Fathers and Early Western Chant, Ashgate, ("Variorum»), Aldershot, 1998.

—, 1994, article "Musik III. Alte Kirche und Mittelalter», in TRE 23, pp. 452-457.

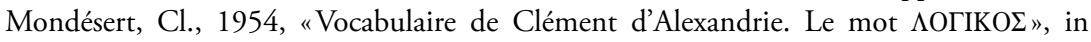
RecSR 42, pp. 258-65.

Naldini, M., 1993, «I miti di Orfeo e di Eracle nell'interpretazione patristica», in SRIC 14, pp. 331-343. 
Quasten, J., 1930, Musik und Gesang in den Kulten der heidnischen Antike und christlichen Frühzeit, Verlag der Aschendorffschen Verlagsbuchhandlung, Münster.

Ricken, Fr., 1967, «Die Logoslehre des Eusebios von Caesarea und der Mittelplatonismus», in Theologie und Philosophie 42, pp. 341-358.

Roessli, J.-M., 2002 «Convergence et divergence dans l'interprétation du mythe d'Orphée, de Clément d'Alexandrie à Eusèbe de Césarée», in RHR 219, pp. 503-513.

Schneider, U., 1999, Theologie als christliche Philosophie, "Zur Bedeutung der biblischen Botschaft im Denken des Clemens von Alexandria», Walter de Gruyter, Berlin-New York.

Skeris, R. A., 1976, XPSMA $\Theta E O Y$, "On the Origins and Theological Interpretation of the Musical Imagery used by the Ecclesiastic Writers of the First three Centuries, with special Reference to the Image of Orpheus », Gebr. Geiselberger ("Musicae sacrae meletemata»), Altötting.

Tabaglio, M., 1999, "La cristianizzazione del mito di Orfeo", in A. M. Babbi (ed.), Le metamorfosi di Orfeo, Fiorini, Verona, pp. 65-82.

Vieillefon, L., 2003, La Figure d'Orphée dans l'Antiquité tardive, «Les mutations d'un mythe : du héros païen au chantre chrétien», De Boccard («De l'archéologie à l'histoire»), Paris. 